\title{
Rubber band-assisted, one-person-operated cold snare polypectomy for colorectal polyps
}

Cold snare polypectomy (CSP) for colorectal polyps has become popular due to its simplicity and safety [1-3]. However, conventional CSP still requires assistants to manipulate the snare. Here, we present the case of a patient with a 5-mm sigmoid colon polyp which was treated by rubber band-assisted, one-person-operated CSP (RAO-CSP) ( Video 1$)$.

RAO-CSP was performed as follows: 1) the endoscope was inserted and placed to capture a target polyp with two rubber-bands attached to the base of the

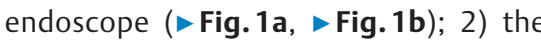
rubber bands were moved to the appropriate position close to the anus and the sheath of the snare was passed through the rubber-band ( $\mathbf{F i g . 2 a , ~} \mathbf{F i g . 2 b}$ ); 3 ) the handle of the snare was fixed to the endoscope axis by clamping it with the rubber bands ( $\mathbf{F i g . 2 c}$ ); 4) the tip of the snare was subsequently introduced into the lesion through the endoscopic channel; 5) the snare was opened around the lesion ( $>$ Fig. 2d); and 6) finally, the snare was closed, and en bloc resection was achieved.

Both snare manipulation and endoscope handling to acquire an appropriate treatment field were performed using the right hand. The distance from the snare to the lesion and the angle of the endoscope were adjusted using the left hand. Fixing the handle of the snare to the endoscope axis with the rubber bands allowed the whole procedure to be performed by only one operator.
One advantage of the RAO-CSP is that it can be applied during colonoscopy screening by one operator without any assistants. Another advantage is that it precludes the effects of variation in assistant skills on the technical outcome of CSP. However, RAO-CSP should only be performed for small polyps. A switch should be made to conventional CSP whenever the operator experiences difficulties with the procedure. Future studies are required to confirm the feasibility and safety of RAO-CSP before widespread use of this technique is adopted in clinical practice.
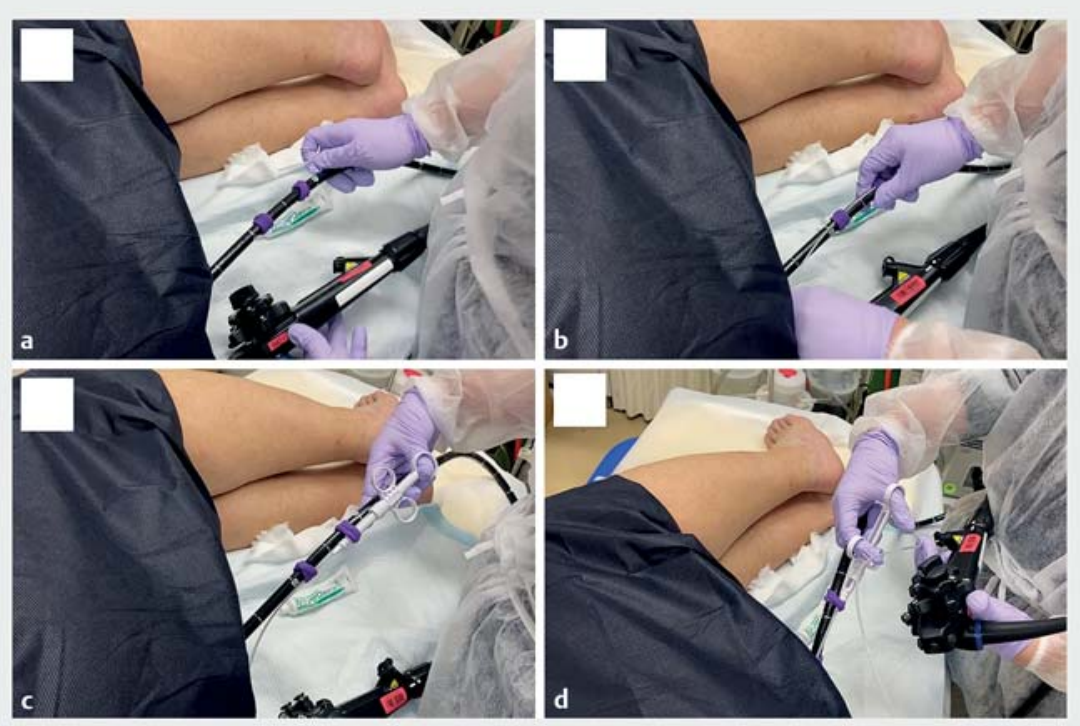

Fig. 2 The protocol for rubber band-assisted, one person-operated cold snare polypectomy (RAO-CSP). a The two rubber bands are appropriately repositioned close to the anus of the patient. $\mathbf{b}$ The snare sheath is passed through the rubber bands. $\mathbf{c}$ The handle of the snare is fixed to the axis of the endoscope by clamping it with a hairband. $\mathbf{d}$ Manipulation of the snare and control of the endoscope are performed using the right hand. 


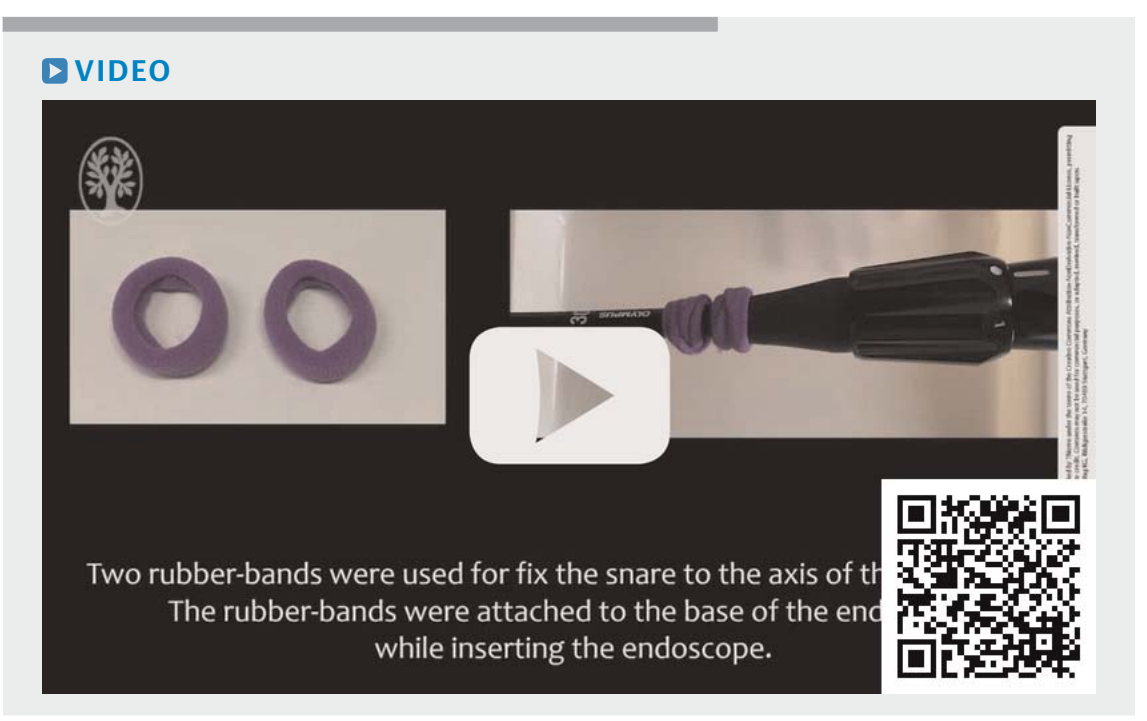

- Video 1 Rubber band-assisted, one-person-operated cold snare polypectomy (RAOCSP) for the treatment of colorectal polyps.

The handle of the snare was fixed to the axis of the endoscope by clamping it with the rubber band. Both snare manipulation and endoscope handling were performed using the right hand. The distance of the snare to the lesion and angle of endoscope were adjusted using the left hand. An en bloc resection of the polyp was successfully achieved.

\section{Bibliography}

Endosc Int Open 2021; 09: E1845-E1846

DOI 10.1055/a-1576-7673

ISSN 2364-3722

(C) 2021. The Author(s).

This is an open access article published by Thieme under the terms of the Creative Commons Attribution-NonDerivativeNonCommercial License, permitting copying and reproduction so long as the original work is given appropriate credit. Contents may not be used for commercial purposes, or adapted, remixed, transformed or built upon. (https:// creativecommons.org/licenses/by-nc-nd/4.0/)

Georg Thieme Verlag KG, Rüdigerstraße 14, 70469 Stuttgart, Germany

\section{$($ ()ㅇㅇㅇ $\odot$}

\section{Competing interests}

Dr. Ihara participated in a study supported of Takeda Pharmaceutical Co., Ltd., belongs to the endowed course supported by the companies mentioned, including Ono Pharmaceutical Co., Ltd., Miyarisan Pharmaceutical Co. Ltd., Sanwa Kagaku Kenkyusho Co., Ltd., Otsuka Pharmaceutical Factory, Inc., Fujifilm Medical Co., Ltd., Termo Corporation, Fancl Corporation, and Ohga Pharmacy, and received a lecture fee from Takeda Pharmaceutical Co. Dr. Ogawa is conducting a joint study with Fancl Corporation and Fujifilm Medical Co., Ltd.

The authors

Misato Esaki ${ }^{1}$, Mitsuru Esaki ${ }^{1,2}$, Kosuke Maehara ${ }^{1}$, Yosuke Minoda ${ }^{1}$, Haruei Ogino ${ }^{1}$, Eikichi Ihara ${ }^{1,3}$, Yoshihiro Ogawa ${ }^{1}$

1 Department of Medicine and Bioregulatory Science, Graduate School of Medical Sciences, Kyushu University, Fukuoka, Japan

2 Division of Gastroenterology and Hepatology, Department of Medicine, Nihon University School of Medicine, Tokyo, Japan

3 Department of Gastroenterology and Metabolism, Graduate School of Medical Sciences, Kyushu University, Fukuoka, Japan

\section{Corresponding author}

\section{Mitsuru Esaki}

Department of Medicine and Bioregulatory Science,, Graduate School of Medical Sciences, Kyushu University, 3-1-1 Maidashi, Higashi-ku, 812-8582 Fukuoka, Japan Fax: +81-92-642-5286

esaki_saiseikai@yahoo.co.jp

\section{References}

[1] de Benito Sanz M, Hernandez L, Garcia Martinez Ml et al. Efficacy and safety of cold versus hot snare polypectomy for small (5-9 mm) colorectal polyps: a multicenter randomized controlled trial. Endoscopy 2020: doi:10.1055/a-1327-8357

[2] Papastergiou V, Paraskeva KD, Fragaki M et al. Cold versus hot endoscopic mucosal resection for nonpedunculated colorectal polyps sized $6-10 \mathrm{~mm}$ : a randomized trial. Endoscopy 2018; 50: 403-411

[3] Ferlitsch M, Moss A, Hassan C et al. Colorectal polypectomy and endoscopic mucosal resection (EMR): European Society of Gastrointestinal Endoscopy (ESGE) Clinical Guideline. Endoscopy 2017; 49: 270-297 